\title{
Radio frequency induced ionized collisional flow model for application at atmospheric pressures
}

\author{
Subrata Roy ${ }^{\text {a) }}$ \\ Computational Plasma Dynamics Laboratory, Mechanical Engineering, Kettering University, Flint, \\ Michigan 48504 \\ Datta Gaitonde \\ Computational Sciences Branch, Air Vehicles Directorate, Air Force Research Laboratory, \\ Wright Patterson AFB, Ohio 45433
}

(Received 15 March 2004; accepted 10 June 2004)

\begin{abstract}
We present the development and application of a versatile finite-element method to discretize direct current and radio frequency (rf) induced plasma-sheath dynamics, using multifluid equations. For the former, argon gas is assumed, and the solution is verified by comparison with a theoretical model obtained from the literature. For rf discharges, partially ionized helium gas is considered between two electrodes coated in a dielectric material. The computed solutions for charge densities, the ion velocity and the neutral gas density and crossflow distributions show expected trends. Specifically, ion and electron number densities at the peak discharge current are compared with published numerical results. The derived electric field is utilized with a simple phenomenological model applicable to the transverse velocity in a one-dimensional situation to predict the anticipated hump in the near wall profile. The next step of extending the model, through future work, to two dimensions and for polyphase supply as implemented in realistic configurations is greatly facilitated by the generality of the chosen finite-element method. () 2004 American Institute of Physics. [DOI: $10.1063 / 1.1778474]$
\end{abstract}

\section{INTRODUCTION}

The advantages of plasma actuators for flow control are being increasingly recognized in the literature in all speed regimes encountered in aerospace application. In addition to the absence of moving parts and rapid switch-on/off capabilities, electromagnetic forces have the potential to apply large forces in a relatively precise manner. Recent experiments have demonstrated striking effects at both low ${ }^{1,2}$ and high speeds. ${ }^{3,4}$ The configurations considered include both volume and surface discharges, at pressures of atmospheric level at low speeds to $O(10)$ Torr at high speeds. While direct current (dc)-based methods are a useful study for energy interactions of an already ionized flow, recent literature ${ }^{5,6}$ shows that energy budgets will depend crucially on dynamic nonequilibrium ionization techniques (the load factor for dc sheath application will be of order 1, far from the most energy efficient Stoletow point, thus unsuitable for ionization purposes). The basic mechanisms inherent in nonequilibrium discharges such as obtained through radio frequency (rf) or microwave excitation have also been utilized for ionization purposes, so as to increase the conductivity of air for further control with ponderomotive forces generated with an imposed magnetic field.

Several different specific configurations have been proposed to exploit the complex interaction between the electric field and the fluid. ${ }^{1,7,8}$ The focus of this effort is on methods that utilize rf or low frequency methods. rf-based methods have seen increasing popularity in recent times. In 1991, an

\footnotetext{
${ }^{a)}$ Author to whom correspondence should be addressed; electronic mail: sroy@kettering.edu
}

ac voltage based method was developed ${ }^{9}$ to produce weakly ionized plasmas which was then utilized to control airfoil flows at low speed (subsonic) flows. ${ }^{10}$ The dynamics of sheath regions under these conditions are fundamentally different and substantially more difficult to simulate because of their unsteady nature. The experimental innovation of rfinduced atmospheric uniform glow discharge ${ }^{1,11}$ (AUGD) shows tremendous potential in the areas of electro gas dynamic flow control and energy management. Figure 1 shows schematics of rf-induced atmospheric glow discharge for electrohydrodynamic (EHD) flow control. The two configurations exert paraelectric and peristaltic flow acceleration, respectively, at low speed with reasonable input power. According to Roth ${ }^{1}$ the former mechanism is associated with plasma acceleration towards an increasing electric field (electrostatic body force) while the latter mechanism is predicated on traveling electrostatic wave (EHD body force) using polyphase power supply. Several papers highlight the theoretical and numerical efforts for this dielectric barrier plasma discharge. ${ }^{2,12,13}$ These efforts span a range of phenomenological to first-principles based methods. However, the causal mechanisms that drive the observed phenomena remain unclear, as is the capability for utilization at higher speeds.

Low-speed flow control typically employs features of the dielectric-barrier discharge to generate a near-surface force that can reattach separated flows. ${ }^{10}$ One electrode is typically exposed to the flow surface, while the other is embedded in the body and displaced a short streamwise distance from it. The electric field generated by the discharge is a consequence of this geometric asymmetry as well as that 


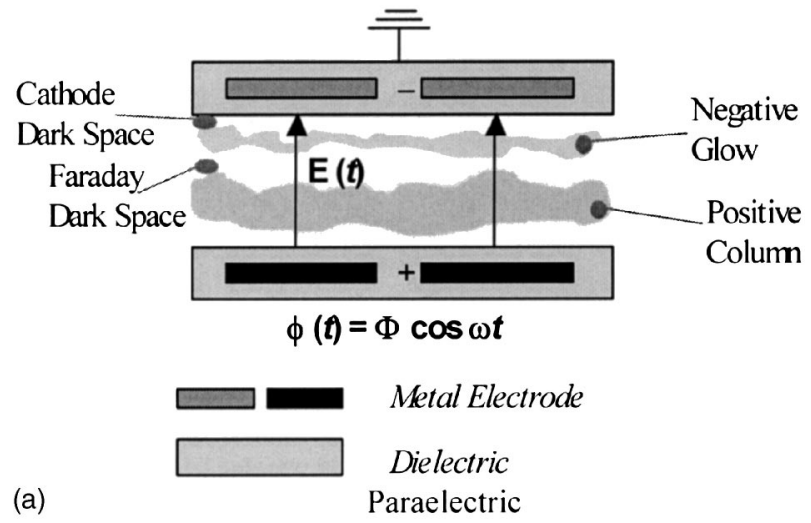

(b)

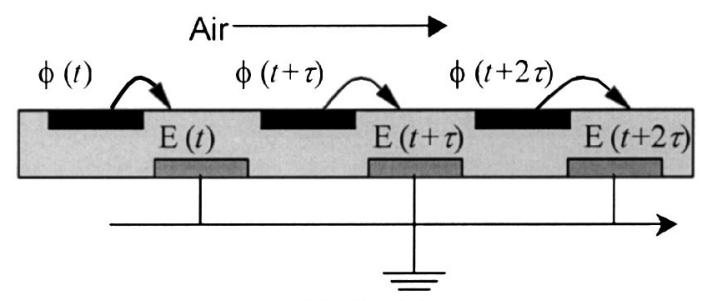

Peristaltic

FIG. 1. rf-induced atmospheric glow discharge for EHD flow control.

associated with the vastly different mobility of the electrons and ions, respectively. The net forces generated by the intermittent discharge induce ion motion, while ion-neutral collision transfer mechanisms generate the desired surface walljet-like effect. The response of the fluid to these forces requires the solution of the full fluid equations past complex configurations under conditions where transition and turbulence are dominant. Consequently, phenomenological models for forces generated by rf discharges have been employed. For example, Shyy, Jayaraman, and Andersson, considers a linear variation of the electric field with a constant charge density and assumptions of the duty cycle to obtain an expression for the force. For further progress in terms of accuracy and fidelity, however, it is essential that the force model be derived from first-principles through a simulation of the elementary mechanisms that yield the discharge. It is precisely the goal of this effort to set the basis for a sophisticated model, with the capability not only to model the physics, but also to do so with a method that is easily extendible to complex configurations.

First-principles approaches at simulation of rf interactions with fluids remain in an early stage of development. Recent experimental and theoretical advances aside, an adequate self-consistent model describing the unsteady dynamics of critical regions of weakly ionized plasma remains a challenge. The numerical model developed in this paper includes consistent calculation for charge and neutral number densities, their momentum dynamics, electric field and potential distribution and is anchored in an efficient, robust, and versatile finite-element approach. For the sake of simplicity, however, we presently focus on helium, the plasma coefficients for which are relatively well known. The methodology is couched in a manner, which can be extended to air with the addition of new mechanisms in the source terms. The intention is to provide a tool to explore flow control con- cepts, develop suitable inputs for traditional fluid dynamics codes based on the Navier-Stokes equations, and to complement experimental efforts in future design and development. The powerful high-fidelity finite-element procedure was adapted from fluid dynamics to overcome the stiffness of the equations generated by multispecies charge separation phenomena. Although the primary focus is on rf discharges, several dc calculations on the traditional physical configuration of near wall electrical discharge to generate ionized gases with a dc voltage placed across two electrodes at either end of a practically evacuated sealed tube was also modeled to provide a baseline for comparison. As the voltage potential is gradually increased, at the breakdown voltage $V_{B}$, the current becomes large enough to produce visible plasma.

\section{MODEL DESCRIPTION}

The basic theoretical details of collisional plasma-sheath with space charge effect for dc and RF power source are adopted from Ref. 15. The model is first verified for a collisionless one-dimensional dc discharge problem for which the normalized equation system and its analytical solution is known. ${ }^{16,17}$ For this case, we assume Boltzmannian distribution of electron density $n_{e}$. The ion density $n_{i}$, ion velocity $V_{i}$, magnitude of the potential $\phi$, and electric field $E$ at steady state are predicted self-consistently from the bulk plasma to the wall using the following normalized equation set:

$$
\begin{aligned}
& \partial_{, x}\left(n_{i} V_{i}\right)=n_{e}=\exp (-\phi), \\
& V_{i} \partial_{, x} V_{i}-E+V_{i} n_{e} / n_{i}=0, \\
& \partial_{, x} E-\kappa^{2}\left(n_{i}-n_{e}\right)=0, \\
& \partial_{, x} \phi-E=0 .
\end{aligned}
$$

Note that the above normalized equations set assumes singly ionized plasma with ionization rate of one. For argon gas, $\kappa^{-1}=4.036 \times 10^{-3}$. The reported solution ${ }^{16}$ for Eqs. (1a)-(1d) is well approximated by the plasma solution for up to $V_{i}<1$.

The equations for the rf-induced glow discharge through dielectric barrier do not utilize the Boltzmann equilibrium assumption for the electrons; hence the electron density is calculated. The model assumes no multiply charged ions and incorporates the following charge and neutral gas continuity equations. Poisson equation is used for calculating electric field and potential distribution.

$$
\begin{aligned}
& \partial_{, t} n_{\alpha}+\partial_{, x}\left(n_{\alpha} V_{\alpha}\right)=n_{e} z \\
& \text { for } \alpha=e, i\left\{\begin{array}{l}
n_{i} V_{i}=n_{i} \mu_{i} E-D_{i} \partial_{, x} n_{i} \\
n_{e} V_{e}=-n_{e} \mu_{e} E-D_{e} \partial_{, x} n_{e},
\end{array}\right. \\
& \partial_{, t} n_{n}+\partial_{, x}\left(n_{n} V_{n z}\right)=-n_{e} z, \\
& \varepsilon \partial_{, x} E-e\left(n_{i}-n_{e}\right)=0, \\
& \partial_{, x} \phi+E=0 .
\end{aligned}
$$

The electron diffusion is obtained from Einstein relation, $D_{e}=\left(T_{e} / e\right) \mu_{e}$, where $T_{e}$ is the energy in electron volts, $e$ is 
the elementary charge, and $\mu_{e}=e /\left(m_{e} \nu_{e h}\right)$ is the mobility of an electron, where $\nu_{e h} \approx 10^{12} / \mathrm{s}$ is the electron-neutral collision frequency. ${ }^{18}$ The ion diffusion $D_{i}=500 \mathrm{~cm}^{2} / \mathrm{s}$ at $300 \mathrm{~K}$, and the ion mobility $\mu_{i}$ is given as ${ }^{19}$

$$
\begin{aligned}
p \mu_{i}= & 8 \times 10^{3}\left[(1-8) \times 10^{-3} E / p\right] \mathrm{cm}^{2} \mathrm{~V}^{-1} \mathrm{~s}^{-1} \mathrm{Torr} \\
& \text { for } E / p \leqslant 25 \mathrm{~V} \mathrm{~cm}^{-1} \mathrm{Torr}^{-1}, \\
p \mu_{i}= & \frac{4.1 \times 10^{4}}{\sqrt{E / p}}\left(1-\frac{27.44}{(E / p)^{3 / 2}}\right) \mathrm{cm}^{2} \mathrm{~V}^{-1} \mathrm{~s}^{-1} \mathrm{Torr} \\
& \text { for } E / p>25 \mathrm{~V} \mathrm{~cm}^{-1} \mathrm{Torr}^{-1} .
\end{aligned}
$$

In Eq. (3), $E$ is the electric field magnitude and $p$ is the pressure. The ionization rate $z$ for helium gas used here is ${ }^{19}$

$$
\begin{aligned}
& z=A \exp \left(\frac{-B}{(E / p)^{0.4}}\right) p \mu_{e} E \mathrm{~s}^{-1} ; \quad A=4.4 \mathrm{~cm}^{-1} \operatorname{Torr}^{-1} \\
& \quad \text { and } B=14[\mathrm{~V} /(\mathrm{cm} \text { Torr })]^{0.4}
\end{aligned}
$$

where $n_{e}$ is the electron number density.

The effect of the excitation on the neutrals is simulated by considering a separate momentum equation for the crosswise neutral gas velocity $V_{n z}$ for this species.

$$
\begin{aligned}
\left(\partial_{, t}+\partial_{, x} V_{n z}\right) V_{n z}= & \left(1 / 2 m_{n} n_{n}\right) \partial_{, x}\left(\varepsilon E^{2}\right)+\left(m_{e} / m_{n}\right) \nu_{e n}\left(V_{e}\right. \\
& \left.-V_{n z}\right)+\left(M / m_{n}\right) \nu_{i n}\left(V_{i}-V_{n z}\right)+S V_{i} / n_{n} .
\end{aligned}
$$

Here, $m_{e}, M$, and $m_{n}$ are mass of electron, ion, and neutral, respectively, $\varepsilon$ is the permittivity, $V_{i}$ and $V_{e}$ are ion and electron velocities, while $\nu_{e n}$ and $\nu_{i n}$ are the electron-neutral and ion-neutral collision frequencies, respectively. The model assumes that $\partial_{, x} \gg \partial_{, z}$, and terms involving the effect of electrons and neutrals are modeled in the standard manner. For practicality, we used the magnitude of $V_{n z}=V_{n x}$ in Eqs. (2b) and (5). In the absence of other external forces, as a first approximation, it is assumed that the hydrodynamic and electrostatic pressure gradients are in approximate equilibrium. ${ }^{1}$ This assumption, although not appropriate at collision rates representative of low pressures, is reasonable at the present study, which considers atmospheric conditions.

\section{NUMERICAL METHOD}

Finite element (FE) techniques are well known for their adaptability to arbitrary multidimensional geometries and boundary conditions. Here, a one-dimensional (1D) finite element formulation is employed to solve Eqs. (1a)-(1d), (2a)(2d), and (5) which may be expressed as $L(\mathbf{U})=0$, where $\mathbf{U}$ contains all state variables, e.g., ion density, ion velocity, and potential, and $L$ is a differential operator. The weak statement associated with a variational integral underlines the development of this numerical algorithm. The physical domain is spatially semidiscretized ( ${ }^{h}$ approximated) using generic computational domain, i.e., the finite element. The state variables are interpolated inside the element, via the trial space FE basis set $N_{k}\left(x_{j}\right)$ that typically contains Chebyshev, Lagrange, or Hermite interpolation polynomials complete to degree $k$ and to dimension $j$. The spatially semidiscrete FE implementation of the weak statement $\mathrm{WS}^{h}$ for $L(\mathbf{U})=0$ leads to

$$
\begin{aligned}
\mathrm{WS}^{h}= & S_{e}\left(\int_{\Omega_{e}} N_{k} L_{e}(\mathbf{U}) d \tau\right) \equiv 0=S_{e}\left(\int_{\Omega_{e}} N_{k} \frac{d \mathbf{U}}{d t}\right. \\
& +\int_{\Omega_{e}} N_{k}(s) d \tau+\int_{\Omega_{e}} \frac{\partial N_{k}}{\partial x_{j}}\left(f_{j}-f_{j}^{\nu}\right)_{e} d \tau \\
& \left.-\oint_{\partial \Omega_{e} \cap \partial \Omega^{h}} N_{k}\left(f_{j}-f_{j}^{\nu}\right)_{e} \hat{n}_{j} d \sigma\right) .
\end{aligned}
$$

$S_{e}$ symbolizes the "assembly operator" carrying local (element $e$ ) matrix coefficients into the global arrays, $s$ is a source term [e.g., $z n_{e}$ in Eq. (1)] and $\mathbf{f}_{j}$ and $\mathbf{f}_{j}^{\nu}$ are convective and disspative flux vectors, respectively, and $\hat{n}$ is the direction normal. Application of Green-Gauss divergence theorem in Eq. (6) yields natural homogenous Neumann boundary conditions. The surface integral in the second line of Eq. (6) contains the (un)known boundary fluxes wherever fixed or flux boundary conditions are enforced.

Independent of the physical dimension of $\Omega$, and for general forms of the flux vectors, the semidiscretized weak statement always yields an ordinary differential equation (ODE) system that is fully discretised using a $\theta$-implicit or $\tau$-step Runge-Kutta type time integration procedure. The terminal ODE is usually solved using a Newton-Raphson scheme for $\mathbf{U}(t)$,

$$
\mathbf{U}_{\tau+1}^{i+1}=\mathbf{U}_{\tau+1}^{i}+\Delta \mathbf{U}^{i}=\mathbf{U}_{\tau}+\sum_{p=0}^{i} \mathbf{U}^{p+1},
$$

where

$$
\Delta \mathbf{U}^{i}=-[\mathbf{M}+\theta \Delta t(\partial \mathbf{R} / \partial \mathbf{U})]^{-1} \mathbf{R}(\mathbf{U}) .
$$

Here, a $\theta$-implicit time marching procedure is employed. In Eq. (7), $\mathbf{M}=S_{e}\left(\mathbf{M}_{e}\right)$ is the "mass" matrix associated with element level interpolation, $\mathbf{R}$ carries the element convection, diffusion and source information. The calculation of the "Jacobian" $\partial \mathbf{R} / \partial \mathbf{U}$ and inversion of the $\mathbf{M}+\theta \Delta t(\partial \mathbf{R} / \partial \mathbf{U})$ matrix with sufficient accuracy is obviously a numerical challenge. However, unlike the traditional finite difference/ volume methods, the present FE algorithm allows one to simulate the system simultaneously without requiring any subiteration for the Poisson solver.

Further details of the code are described in Refs. 20 and 21. The solution is convergent at any given timestep when the maximum value of the residual relative norm for each of the state variable becomes smaller than a chosen convergence criterion of $10^{-4}$. It is declared steady state as the transient features die down and the solution at any time step converges to the $10^{-5}$ within the first iteration.

\section{RESULTS AND DISCUSSION}

The combined plasma-sheath dynamics is modeled on a one-dimensional geometry. Equation sets (1a)-(1d), (2a)(2d), and (5) are solved using second-order time accurate implicit $(\theta=1)$ finite element method over a computational domain $\left(x: 0, x_{w}\right)$. Two cases are simulated for verification and benchmarking of the algorithm with available results. 

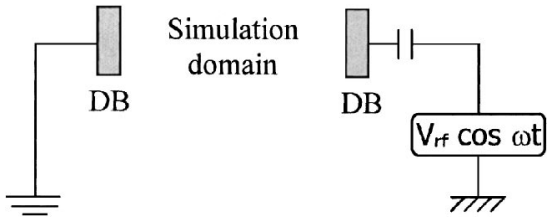

FIG. 2. Schematic of the simulation domain between two insulated electrodes for rf-induced dielectric barrier (DB) discharge.

The first considers the dc discharge, for which the Sternberg solution ${ }^{16}$ is employed in the collisionless assumption. The ion density and velocity at the center of the plasma $(x=0)$ were set to be 1 and 0 , respectively, while the electric field and potential of the bulk were both set to be zero. The schematic of the second case shown in Fig. 2 considers the rf discharge through the dielectric barrier (DB). The left electrode is grounded while a rf alternating frequency is $50 \mathrm{kHz}$ with rms potential of $1.2 \mathrm{kV}$ is imposed at the right electrode. The electrodes are coated with $0.6 \mathrm{~mm}$ alumina and are kept at $5 \mathrm{~mm}$ distance from each other. Electrons are assumed to be isothermal at the boundary and maintained at $1 \mathrm{eV}(\sim 11600 \mathrm{~K})$ while the ions are cold $(300 \mathrm{~K})$ at 300 torr. All other boundary conditions are maintained at zero flux, i.e., homogeneous Neumann boundary conditions are applied. The solutions are verified by comparison with the reported results of Massines et al. ${ }^{13}$ The results are then employed to explore the enhancement of near wall neutral velocity. This model presently does not include secondary emission.

\section{A. dc discharge simulations}

We first solved the dc plasma-wall equation on 100 uniform quadratic elements (201 equidistant points). Sternberg and Godyak ${ }^{16}$ derived the analytical solutions for this problem from the center of plasma till the region where ion Bohm velocity is reached. Figures 3(a)-3(d) show excellent com- parison of the simulation prediction for ion number density, velocity, electric field, and potential distributions for $\kappa^{2}$ $=61390$ with the published results ${ }^{16}$ for a normalized wall potential of 50. The wall is located at a normalized $x_{w}$ $=0.71147$. The solution from Ref. 16 is plotted on every $3 \%$ distance of the domain for visual clarity. Figure 3 also shows the effect of nondimensional permittivity $\kappa^{-1}$ on the state variables. As $\kappa^{2}$ increases from 6139 to 61390 , the ion density decreases from 0.2 to 0.05 in Fig. 3(a) and the ion velocity increases from 3 to 10 in Fig. 3(b). The effect is evident in Figs. 3(c) and 3(d) showing nearly tenfold increase in the magnitude of the wall potential and electric field, respectively. Figure 3(a) also plots the growth of the sheath as the Boltzmannian electron number density bifurcates near $(\pi / 2$ -1) from the ion number density solution for $\kappa^{2}=61390$. The location of this sheath edge is also noticeable for ion velocity, potential, and electric field distribution in Figs. 3(b)-3(d).

\section{B. rf discharge simulations}

For benchmarking with the solution reported by Massines et al. ${ }^{13}$ all numerical results presented here are at $9.3 \mu \mathrm{s}$ where the discharge current is the maximum. The mesh consists of 200 equal length 1D quadratic finite elements (i.e., 401 nodes). The algorithm is spatially third-order accurate. The applied rms voltage and forcing frequency are $1.2 \mathrm{kV}$ and $50 \mathrm{kHz}$ respectively.

Numerical prediction for the ion number density distribution plotted in Fig. 4(a) using the finite element procedure demonstrates noticeable similarity with previously reported results. ${ }^{13}$ The peak of ion number density near the instantaneous cathode is $6 \times 10^{17} \mathrm{~m}^{-3}$ which is $16 \%$ higher than that previously reported. Corresponding electron number density shown in Fig. 4(b) also compares within $+18 \%$ disparity with published results ${ }^{13}$ in charge prediction. Evidently, electron number density starts to decrease rapidly near the plasma-
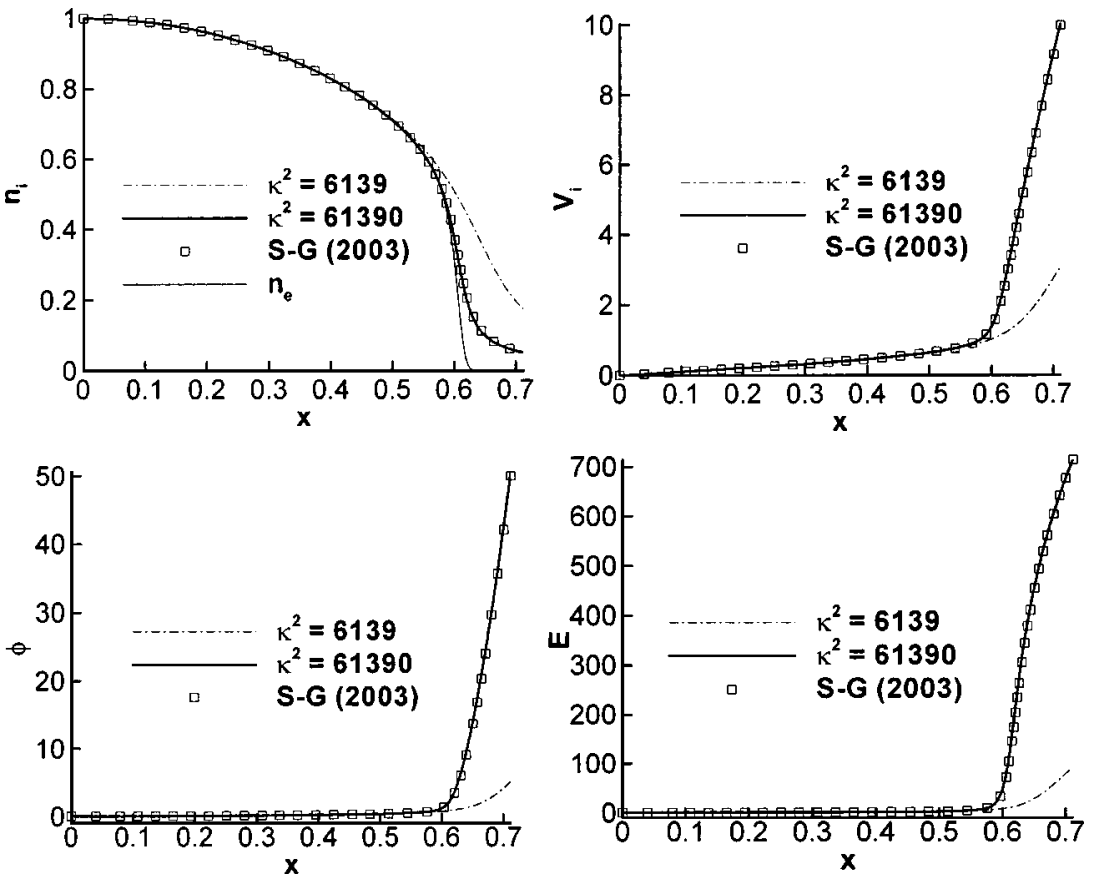

FIG. 3. dc sheath solution compared with Ref. 16: (a) ion number density, (b) ion velocity, (c) magnitude of the potential, and (d) electric field distribution. 


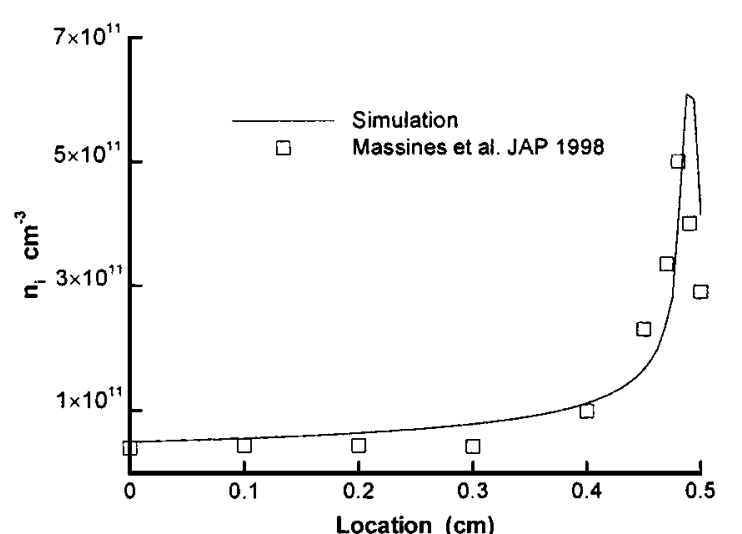

(a)

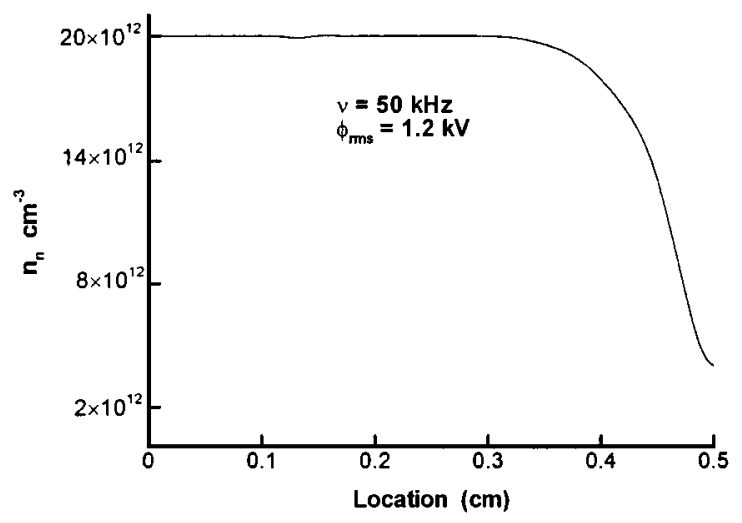

(c)
Ion density comparison

Neutral density distribution

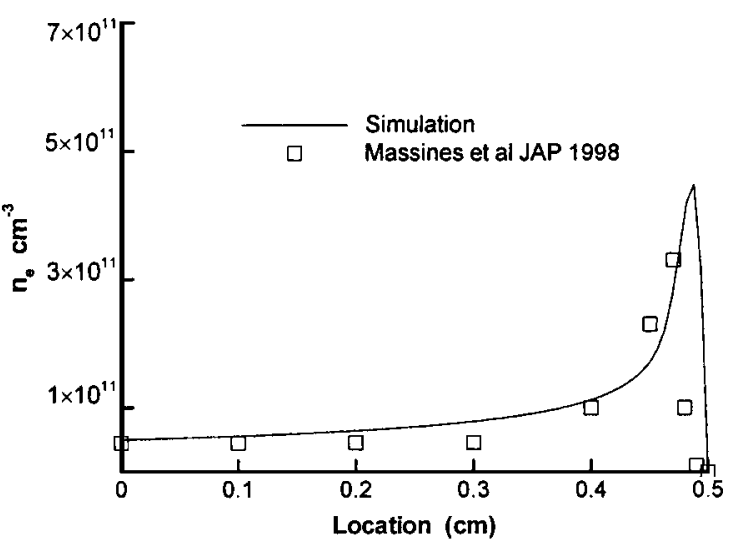

(b)

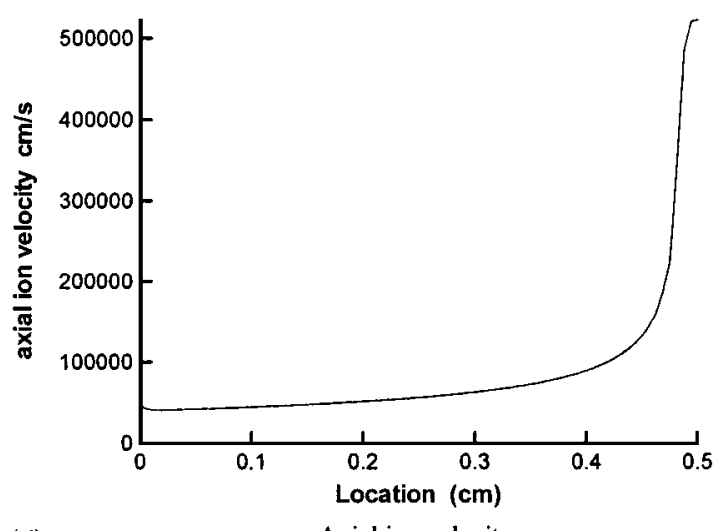

(d)

Axial ion velocity

FIG. 4. rf glow discharge solution at maximum discharge current (a) ion number density, (b) electron number density, (c) neutral density distribution in $\mathrm{cm}^{-3}$, and (d) axial ion velocity in $\mathrm{cm} / \mathrm{s}$.

sheath boundary and becomes almost zero at the instantaneous cathode. This is expected due to the build up of a strong negative potential at the wall, causing electrons to be expelled from the presheath-sheath region and only suprathermal electrons to be able to overcome the potential barrier and cross over to the wall. The number of suprathermal electron is always small in any given plasma. Figure 4(c) shows the neutral number density decay due to the ionization process in rf glow discharge. Noticeably the neutral density reduces by an order of magnitude in the region where maximum charge is produced. However, the gas density does not increase as the charge density decreases primarily due to the absence of recombination in the present model [see Eq. (2b)]. The dielectric does not allow charges to move along it, so recombination may be delayed. The computed axial ion velocity shown in Fig. 4(d) shows a sharp increase near the cathode fall region crossing the Bohm velocity $(\sim 4000 \mathrm{~m} / \mathrm{s}$ near the peak of electron density [Fig. 4(b)].

The computed electric field $E=-\partial_{, x} \varphi$ is plotted in Fig. 5 and shows high positive gradients near both electrodes. Interestingly, the field becomes negative predicting a sharp drop of potential near the anode (fall) and the same effect will be expected near the cathode. This phenomenon is due to electron waves and is well explained by Raizer et al. ${ }^{22}$

For exploratory purposes, the neutral gas flow in the crosswise direction is also predicted using the assumptions noted earlier. Although this assumption is based on relatively simplistic reasoning and apparently valid only for 1D situations $^{2}$ it implicitly factors the charge distribution through the electric field gradient and serves as a simple method of extracting a body force from the computed electric field. Within these limitations, the crossflow gas velocity calculated using Eq. (5) is plotted in Fig. 6(a) shows reasonable trend in comparison to the test data in Fig. 6(b) reported by Roth. ${ }^{1}$ The purpose of the figure is to show the similarity of computed and experimental profiles demonstrating the

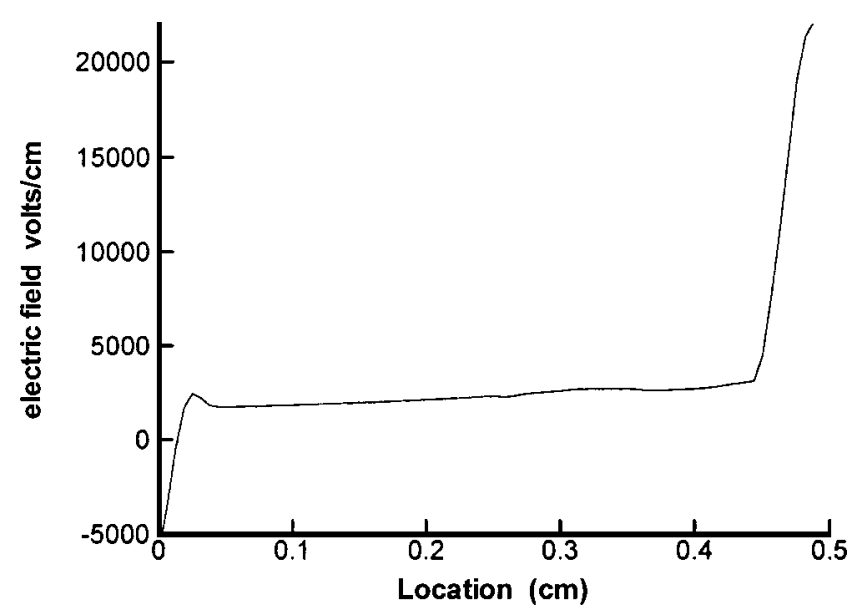

FIG. 5. Electric field distribution between the electrodes at the peak discharge current. 

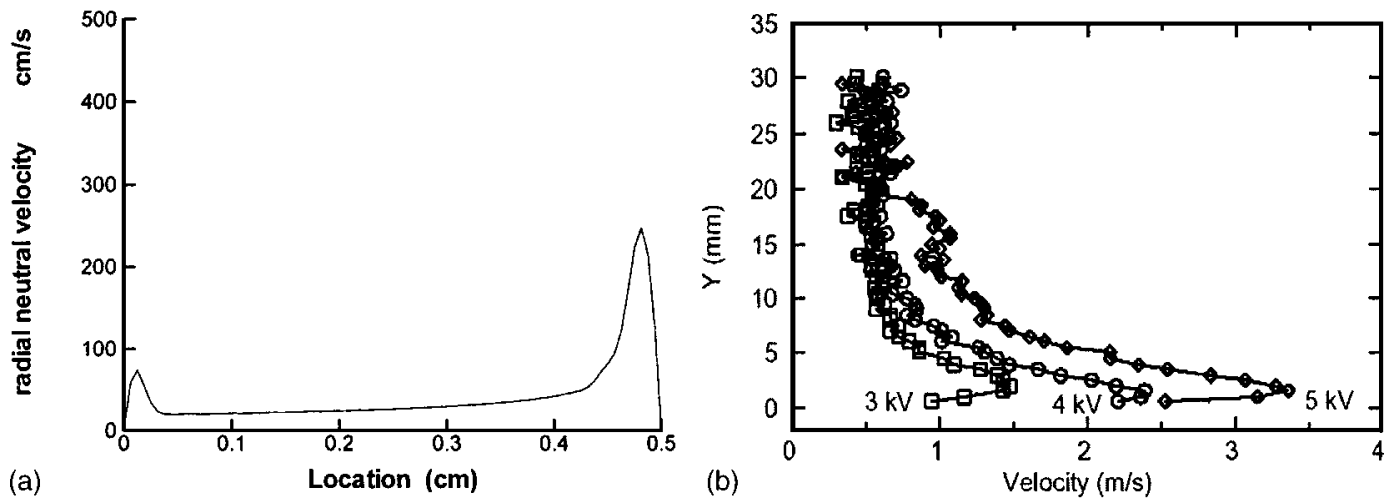

FIG. 6. (a) Calculated magnitude of crosswise component of the gas velocity distribution, (b) experimental velocity distribution normal to the surface (Roth, Ref.1).

augmentation of near-wall momentum to yield a wall-jet-like structure through a first-principles approach. The similar quantitative values of the maxima are likely fortuitous, since they consider different fluids under different physical setups. The large electric field gradients near each electrode are associated with charge separation consistent with the different local ion and electron densities. The net force, related to the product of this charge and the electric field, peaks near each electrode. The computed crosswind thus shows two peaks, one near each electrode. However, due to higher electric field the velocity near the instantaneous anode is $1 / 5$ th of that near the cathode, Fig. 6(a). The parameters chosen for the simulation yield relatively low-induced velocities. Experimental evidence ${ }^{2}$ suggests that proper placement of such control devices in critical fluid dynamic regions can further leverage the EHD effect by influencing the bifurcation, for example, through suppression of separation, and a fundamental change in the topology. Nonetheless, methods of increasing the induced velocity must be explored in the context of scalability issues, which remain to be resolved.

\section{CONCLUSIONS}

A finite element based formulation of plasma-fluid interactions is given for a partially ionized plasma using the multicomponent fluid equation. The model is applied for simulating atmospheric RF glow discharge for partially ionized helium gas between two electrodes. The computed solutions for charge densities, the ion velocity and the neutral gas density and crossflow distributions show anticipated trends. Specifically, ion and electron number densities at the peak discharge current are compared with published numerical results. The electric field driven radial neutral gas velocity compares well with available experimental data. Future research goals include extension to multidimensional configurations, with emphasis on simulation of surface rf discharges, identification of critical physical processes including recombination and secondary emission in rf-based EHD, development of a theoretical model for air of suitable complexity and finally implementation into a powerful, robust and efficient numerical tool. The application of the methodology in understanding the relative importance of Lorentzian momentum transfer versus Joule heating will be particularly useful in determining the effectiveness of rf-based plasma flow con- trol at higher speeds, where compressibility is important. The present effort also sets the stage for exploration in the higherfrequency range encompassing the microwave regime, where substantially higher power levels may be brought to bear.

\section{ACKNOWLEDGMENTS}

S.R.'s work was partially supported by the Air Force Research Laboratory Contract No. F33615-98-D-3210 and the National Academy of Science NRC/AFOSR research fellowship during the summer of 2003. The authors acknowledge many thoughtful discussions about dc sheath with Natalia Sternberg.

${ }^{1}$ J. R. Roth, Phys. Plasmas 10, 2117 (2003).

${ }^{2}$ C. L. Enloe, T. E. McLaughlin, R. D. VanDyken, K. D. Kachner, E. J. Jumper, and T. C. Corke, 41st AIAA Aerospace Sciences Meeting, 2003.

${ }^{3}$ R. B. Miles, AIAA Paper No. 2000-2324 (2000).

${ }^{4}$ S. B. Leonov and V. A. Bityurin, AIAA-2002-5209 (2002).

${ }^{5}$ I. V. Adamovich, V. V. Subramaniam, J. W. Rich, and S. O. Macheret, AIAA J. 36, 816 (1998).

${ }^{6}$ S. O. Macheret, M. N. Shneider, and R. B. Miles, J. Propul. Power 18, 424 (2002).

${ }^{7}$ E. P. Gurijanov and P. T. Harsha, AIAA Paper No. 96-4609 (1996).

${ }^{8}$ Y. C. Ganiev, V. P. Gordeev, A. V. Krasilnikov, V. I. Lagutin, V. N. Otmennikov, and A. V. Panasenko, J. Thermophys. Heat Transfer 14, 10 (2000).

${ }^{9}$ N. Kanda, M. Kogoma, H. Jinno, H. Uchiyama, and S. Okazaki, Proceedings of 10th Symposium on Plasma Chem. 1991.

${ }^{10}$ T. C. Corke, E. J. Jumper, M. L. Post, D. Orlov, and T. E. McLaughlin, 40th AIAA Aerospace Sciences Meeting, 2002.

${ }^{11}$ G. A. Hebner, J. T. Verdeyen, and M. J. Kushner, J. Appl. Phys. 63, 2226 (1988).

${ }^{12}$ W. Shyy, B. Jayaraman, and A. Andersson, J. Appl. Phys. 92, 6434 (2002).

${ }^{13}$ F. Massines, A. Rabehi, P. Decomps, R. B. Gadri, P. Ségur, and C. Mayoux, J. Appl. Phys. 83, 2950 (1998).

${ }^{14}$ M. Mitchner and C. H. Kruger, Partially Ionized Gases (WileyInterscience, New York, 1973).

${ }^{15}$ S. Roy, B. P. Pandey, J. Poggie, and D. Gaitonde, Phys. Plasmas 10, 2578 (2003).

${ }^{16}$ N. Sternberg and V. A. Godyak, IEEE Trans. Plasma Sci. 31, 665 (2003).

${ }^{17}$ J. Poggie and N. Sternberg, AIAA-2004-0177 (2004).

${ }^{18}$ K. Akhtar, J. E. Scharer, S. M. Tysk, and E. Kho, Rev. Sci. Instrum. 74, 996 (2003).

${ }^{19}$ L. Ward, J. Appl. Phys. 33, 2789 (1962).

${ }^{20}$ S. Roy and B. P. Pandey, J. Propul. Power 19, 964 (2003).

${ }^{21}$ D. Balagangadhar and S. Roy, Comput. Methods Appl. Mech. Eng. 190, 5465 (2001).

${ }^{22}$ Y. P. Raizer, M. N. Shneider, and N. A. Yatsenko, Radio-Frequency Capacitive Discharges (CRC, London, 1995), p. 292. 